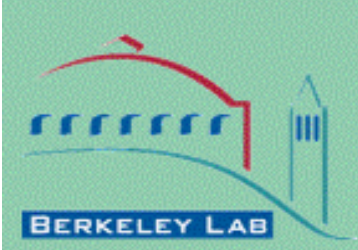

\section{ERNEST ORLANDO LAWRENCE \\ BERKELEY NATIONAL LABORATORY}

\author{
Paul Sheaffer*, Aimee McKane**, \\ Vestal Tutterow***, Ryan Crane****
}

*Resource Dynamics Corporation, **Lawrence Berkeley National Laboratory, ***Alliance to Save Energy, ****American Society of Mechanical Engineers

\section{Environmental Energy \\ Technologies Division}

August 2009

This manuscript has been authored by an author at Lawrence Berkeley National Laboratory under Contract No. DE-AC02-05CH11231 with the U.S.Department of Energy. The U.S. Government retains, and the publisher, by accepting the article for publication, acknowledges, that the U.S. Government retains a non-exclusive, paid-up, irrevocable, world-wide license to publish or reproduce the published form of this manuscript, or allow others to do so, for U.S. Government purposes. 



\section{Disclaimer}

This document was prepared as an account of work sponsored by the United States Government. While this document is believed to contain correct information, neither the United States Government nor any agency thereof, nor The Regents of the University of California, nor any of their employees, makes any warranty, express or implied, or assumes any legal responsibility for the accuracy, completeness, or usefulness of any information, apparatus, product, or process disclosed, or represents that its use would not infringe privately owned rights. Reference herein to any specific commercial product, process, or service by its trade name, trademark, manufacturer, or otherwise, does not necessarily constitute or imply its endorsement, recommendation, or favoring by the United States Government or any agency thereof, or The Regents of the University of California. The views and opinions of authors expressed herein do not necessarily state or reflect those of the United States Government or any agency thereof, or The Regents of the University of California.

Ernest Orlando Lawrence Berkeley National Laboratory is an equal opportunity employer. 



\title{
System Assessment Standards: Defining the Market for Industrial Energy Assessments
}

\author{
Paul Sheaffer, Resource Dynamics Corporation \\ Aimee McKane, Lawrence Berkeley National Laboratory \\ Vestal Tutterow, Alliance to Save Energy \\ Ryan Crane, American Society of Mechanical Engineers
}

\begin{abstract}
Improved efficiency of industrial systems (e.g., compressed air or steam) contributes to a manufacturing facility's bottom line, improves reliability, and better utilizes assets. Despite these advantages, many industrial facilities continue to have unrealized system optimization potential. A barrier to realizing this potential is the lack of market definition for system energy efficiency assessment services, creating problems for both service providers in establishing market value for their services and for consumers in determining the relative quality of these system assessment services.

On August 19, 2008, the American Society of Mechanical Engineers (ASME) issued four new draft Standards for trial use that are designed to raise the bar and define the market for these services. These draft Standards set the requirements for conducting an energy assessment at an industrial facility for four different system types: compressed air, process heating, pumping, and steam. The Standards address topics such as organizing and conducting assessments; analyzing the data collected; and reporting and documentation.

This paper addresses both the issues and challenges in developing the Standards and the accompanying Guidance Documents, as well as the result of field testing by industrial facilities, consultants, and utilities during the trial use period that ended in January, 2009. These Standards will be revised and released by ASME for public review, and subsequently submitted for approval as American National Standards for publication in late 2009. Plans for a related activity to establish a professional-level program to certify practitioners in the area of system assessments, opportunities to integrate the ASME Standards with related work on industrial energy efficiency, as well as plans to expand the system assessment Standard portfolio are also discussed.
\end{abstract}

\section{Background}

System assessments collect and analyze information on industrial system design, operation, energy use, and performance data. This information is used to identify energy efficiency improvements that will optimize system energy use or performance. An assessment may also include additional recommendations for improving resource utilization, reducing per unit production cost, and improving environmental performance related to the assessed system(s). As part of this effort, accompanying Guidance Documents, which provide technical background and application details for utilizing the Standards, were developed for each of the four Standards.

The Standards set the requirements for conducting and reporting the results of an assessment that considers the entire system, from energy inputs to the work performed as the 
result of these inputs. An assessment complying with the Standards need not address each individual system component or subsystem within an industrial facility with equal weight, however, it must be sufficiently comprehensive to identify the major energy efficiency opportunities for improving the overall energy performance of the system. Figure 1 shows the overall assessment process as presented in ASME EA-1-Draft - Energy Assessment for Process Heating Systems.

Figure 1. Assessment Process - Process Heating Systems

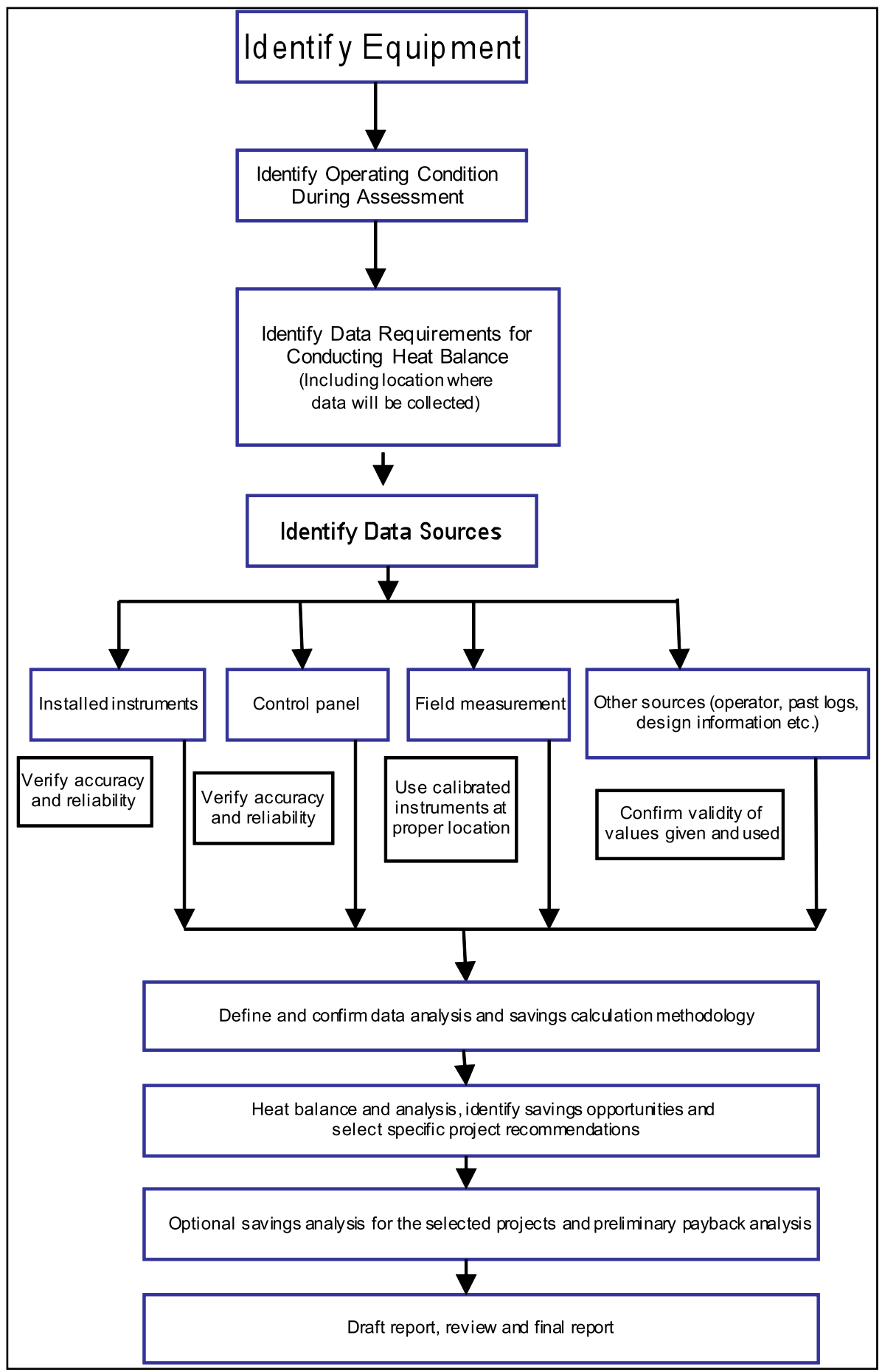




\section{Building Blocks for the Standards}

The United States Department of Energy's Industrial Technologies Program (DOE/ITP) has a well-developed portfolio of system optimization resources and training for industrial motor, compressed air, fan, pump, steam, and process heating systems, which includes system assessment software designed to help consultants and plant personnel quickly identify energy savings opportunities. To ensure proper use of the system assessment software tools, a Qualified Specialist program was developed in 2000 to train and recognize individuals for their skills in each application. Equipment suppliers, consultants, and highly skilled end-users experienced in system optimization in their area of specialty are trained, take an examination, and are then certified. The DOE Qualified Specialist training program includes 2-3 days of focused training on application of a system assessment software tool, followed by a rigorous qualifying examination that tests a candidate's ability to apply the software in conducting system assessments. DOE/ITP has also established a protocol for conducting basic system assessments. Other building blocks include efforts of organizations like the Compressed Air Challenge ${ }^{\mathrm{TM}}$, Pump Systems Matter ${ }^{\mathrm{TM}}$ (developed with support from the Hydraulic Institute), and resources and tools developed at the State and Regional level, such as those developed by Focus on Energy in Wisconsin and the Industrial Energy Efficiency Alliance in the Pacific Northwest. All of these efforts are used as inputs for the development of the Standards and Guidance Documents.

The decision to develop system assessment Standards emerged from work on Superior Energy Performance (SEP), an initiative guided by the U.S. Council for Energy-Efficient Manufacturing, a voluntary partnership between US industry, government and other organizations. The application of the assessment Standards assist plant personnel in identifying cost-effective projects that often have limited capital requirements, and are a key contribution to the framework for assisting US industry to meet the energy intensity improvement criteria of Superior Energy Performance. ${ }^{1}$

\section{Standards Development Process and Project Teams}

The Standards development process has followed the ANSI-accredited procedures for ASME Codes and Standards Development Committees, which establishes consensus-building and has procedures for obtaining public comment. Four Project Teams comprised of 10-15 members from a cross section of industry developed the draft Standards and Guidance Documents. DOE/ITP is supporting a core of two DOE System Experts and a Project Team Coordinator for each Team, with the remaining members drawn from industry, utilities, consultants, national laboratories, equipment manufacturers, and energy efficiency organizations, all with extensive experience in system energy efficiency. Close coordination was achieved through participation by members of the Compressed Air Challenge ${ }^{\mathrm{TM}}$ and Pump Systems Matter ${ }^{\mathrm{TM}}$. An ASME Energy Assessment Consensus Committee oversees the development of all four Standards. Draft Standards for Trial Use were completed in late 2008, and each of the draft Standards was introduced to the respective systems assessment community during a series of webcasts. Comments from the trial use period were being addressed in spring 2009.

\footnotetext{
${ }^{1}$ http://www.superiorenergyperformance.net/
} 
Revisions were made to the draft Standards and Guidance Documents based on the comments from the trial use period. Then the ASME Energy Assessment Consensus Committee will ballot the Standards and they made available by ASME during a public comment period prior to publication.

\section{Assessment Standards}

Assessment activities include but are not limited to engaging facility personnel and providing information about the assessment process; collecting and analyzing data on system design, operation, energy use, and performance; identifying energy performance improvement opportunities; and making recommendations for system improvement and energy-saving project implementation in a written report. The assessment report documents system design; quantifies energy operation and performance data; documents the assessment process; shows results and gives recommendations and savings projections; and improves the plant or facility personnel's understanding of system energy use and operation.

Each of the assessment Standards required a slightly different approach because each of the systems has different characteristics. As an example, a facility could have hundreds of pumping systems, while most facilities only have one compressed air system. Highlights of the approaches used for each Standards are described below.

\section{Pumping Systems}

This Standard differentiates between and has requirements for three types of assessments. The Level 1 (prescreening) assessment is a qualitative investigation that is intended to determine the magnitude of energy optimization potential and therefore determine the necessity for a Level 2 or Level 3 assessment. The Level 1 assessment is used to identify specific systems for further analysis. The Level 2 assessment is a quantitative (measurement-based) investigation meant to determine the energy savings potential for at least one operating condition. The measurements usually cover a limited amount of time, thus giving a snapshot of the operating conditions at the time of measurement. Level 2 assessment are used for systems with little or no variability. The Level 3 assessment is also a quantitative investigation, requiring measurements taken over an extended period of time sufficient to develop a system load profile. This activity is usually associated with more extensive use of in-situ monitoring to ensure that the operating conditions can be accurately determined at the various duty points.

All pumping system assessments start with a Level 1 assessment. During this prescreening, the pumping systems that will undergo further investigation are identified and selected. The outcome of the prescreening process shall be the selection of the best candidates, typically those with significant energy savings potential, for more in depth analysis (Level 2 or Level 3 assessment). The assessment team determines which systems require a Level 2 or 3 assessment based on system characteristics.

The pumping assessment is based on comparing an existing system with an ideal system that uses the optimal amount of energy required to perform the necessary system functions. The standard also has requirements to analyze reducing system head, reducing system flow rate, and ensuring that components are operating close to their best efficiency point.

Pump systems are often a subsystem of a large system (e.g., a chiller system). This

presents a challenge on where to draw the boundary around the system that is being assessed. 
The pump Standard includes requirements for having a clear understanding of how the larger system is affected by changes made to the pumping system. That Standard states that in such cases it may be necessary to connect with persons with knowledge about the larger system, to determine the constraints the larger system puts on potential modifications to the pumping system.

\section{Process Heating Systems}

Like with pumping systems, an industrial plant could have many process heating systems. The process heating has an activity to develop a preliminary list of systems for the assessment. Systems are divided into three categories: 1) those worthy of further analysis, 2) possible candidates for analysis after higher priority opportunities are dealt with and 3) systems unsuitable for assessment. The selection is based on factors such as energy use, applicability, past experience with application of the identified opportunities, current and future operations, and considerations for product quality, safety and equipment life.

The process heating Standard analyzes systems by performing an energy balance (and material balance, when appropriate) based on the collected data to compare the results of this energy balance with industry-accepted benchmarks, determining areas where energy efficiency could be improved, and identifies ways improvements could be obtained.

\section{Steam Systems}

Most plants have only one steam system, so no system prescreening is required in the steam Standard. There is a requirement that the Standard could be used for an individual steam system in an exceptionally large facility that had more than one steam system.

An important approach in the steam Standard relates to over-the-fence supply or demand. If an energy stream derives from or is directed to an adjacent system (possibly in an adjacent energy-supplying or receiving facility) then the details of the energy streams (e.g. electricity, steam, natural gas) need to be considered part of the assessment of the target system.

The steam system Standard analyzes systems by performing a mass and energy balance based on collected data, and this process identifies areas where energy efficiency could be improved.

\section{Compressed Air Systems}

Most plants have only one compressed air system, so no prescreening is required. However, compressed air systems are generally quite complex and have supply, transmission, and demand subsystems, all which must be included as part of the assessment. Optimizing a compressed air system by performing a system assessment generally goes beyond just addressing efficiency issues. Because of this, the compressed air Standard has requirements for assessment activities that lead to energy efficiency gains, but also contains non-required activities that address other performance issues. The compressed air Standard includes these activities in matrices in two appendices. Like the steam Standard, the compressed air Standard has requirements for addressing over-the-fence supply and shared demand among multiple facilities. 


\section{Lessons Learned During the Pilot Period}

During the trial period, the draft Standards were tested at five plants in Texas as part of an ITP/Texas Industries of the Future-led effort. In addition, feedback was obtained from tests in other facilities, and comments were also obtained from "desk reviews" by various parties, including a coordinated review by the Consortium for Energy Efficiency members. The Texas pilots also included implementation of ANSI MSE 2000:2008, the current national energy management standard. Generally, the feedback from the trial period was good - the standards worked well when applied at these facilities.

Significant comments from the trial use period are described below.

\section{Adding Cost Estimates to Efficiency Recommendations}

The draft Standards do not provide requirements to develop implementation costs for the efficiency recommendations developed during the assessment process. The draft Standards include an optional task to develop screening or feasibility estimates of these costs. This issue was debated a length during the development process by the Project Teams. While recognizing an organization's need for cost estimates in determining whether to proceed with an energy efficiency recommendation, the Project Teams of experts determined that these estimates are typically initiated by the plant personnel participating as members of the team as part of a postassessment exercise. In many cases, developing high-quality implementation costs would require a separate effort that could involve a complex engineering study. For these reasons, implementation costs will remain an optional activity.

\section{Make Certain Sections More Consistent with all Four Standards}

As the result of comments, the need for greater consistency was identified for two sections: 1) Organizing the Assessment and 2) Report and Documentation. The final drafts will make these sections more consistent. This will make is easier to implement multiple Standards in a given plant.

\section{Moving Management and Other Issues to Guidance Documents or Non-mandatory Appendices}

Some users commented that the Standards included too many requirements on management issues, such as formal requirements for the composition of the assessment team members, which made the standards cumbersome to use. While preserving the concept of forming a team, much of the detailed material will be provided in the Guidance Document.

\section{Making Reporting Requirements More Flexible}

Some pilot-period testers felt the requirements for the assessment reports were too prescriptive. The revised draft Standards will make the requirements more flexible, although there will be many required items that must still remain in the report to ensure the assessments are comprehensive. 


\section{Offering Different Levels of Assessments}

Some users requested that the Standards address more than one level of assessment (e.g., a walk-through type assessment versus a comprehensive systems assessment). A primary goal behind the development of these Standards is to provide a consistent framework for conducting comprehensive assessments of industrial systems that will help define the market for both users and providers of these services. Efforts are being taken during the revision process to ensure that the Standards are applicable to all types of systems in all sizes of organizations, however assessments must address the entire system and should remain comprehensive. These "walkthrough" type assessments are addressed through protocols developed by other entities. ${ }^{2}$

\section{Addressing Over the Fence Supply, Multiple Owners of Demand, and Systems as Part of Bigger System}

Defining the parameters of the system to be assessed proved to be more difficult than originally anticipated and adjustments are being made to the requirements of the Standards. In some cases, the supply (e.g., steam or compressed air) may be purchased from another entity. In other cases, part of the system demand may be in another facility that is not part of the assessment boundaries. Another problem is when the system being assessed is part of a larger system or process (e.g., a pump system within a chiller system). Changes are being made to the Standards and Guidance Documents to better define what should be included in an assessment.

\section{Related Activities}

A number of related initiatives are currently underway that support the Standards and the industrial energy efficiency goals of the SEP partnership, including:

- International energy management standard, ISO 50001, which is expected to replace ANSI MSE 2000:2008 as the central element of the SEP. Publication of ISO 50001 is anticipated for early 2011. The standard will establish a framework for industrial plants or entire companies to manage all aspects of procurement and use of energy. DOE/ITP is supporting ANSI's U.S. Technical Advisory Group (TAG) and ANSI's role as Secretariat of ISO Project Committee $242 .^{3}$

- Measurement and Verification (M\&V) protocol to validate conformance with ISO 50001 and energy intensity improvements to meet SEP program requirements.

- An initiative to train and certify individuals as Certified Practitioners to assist plants in 1) implementation of ISO 50001 or 2) in one of the four ASME System Assessment Standards. Professional certification will also be offered for SEP Validation Specialists to evaluate conformance with ISO 50001 and the SEP program requirements through the $\mathrm{M} \& \mathrm{~V}$ protocol.

\footnotetext{
${ }^{2}$ Examples: Compressed Air Challenge ${ }^{\mathrm{TM}}$ Levels of Assessment, Bonneville Power Authority Industrial Audit Guide, Pump Systems Matter ${ }^{\mathrm{TM}}$ and BC Hydro Pump System Basic Assessment Guide

${ }^{3}$ More information on ISO 50001 and the role of energy management standards is included in a related 2009 ACEEE Summer Study paper Thinking Globally” How ISO 50001- Energy Management can make industrial energy efficiency standard practice
} 
- End-user awareness training to educate plant personnel on the SEP program requirements, ISO 50001, system assessment Standards, and the M\&V protocol.

These activities will provide industrial facilities with a road map for achieving continual improvement in energy efficiency while maintaining competitiveness. SEP builds upon more than a decade of DOE/ITP experience in conducting system assessments, while expanding the opportunities for energy savings to include the entire industrial facility. Through its reliance on consensus standards and a certification process, SEP seeks to provide market value for these efforts. Companies using the resources will increase the transparency of their efforts to reduce energy consumption. The system assessment Standards play a key role in the overall effort by providing a clear path forward to identify energy savings opportunities.

\section{Professional-Level Program to Certify Practitioners in System Assessment Standards}

In order to provide market recognition of individuals with the necessary skills to properly apply the system assessment Standards and to help potential users of these services in locating qualified individuals, the development of a program to establish Certified Practitioners (CPs) will be pursued. This will consist of a professional credentialing program with certification to verify the competency of users of the Standards. It should be noted that use of a CP will not be a requirement for compliance with the system assessment Standards - the credential is designed to aid plants in locating outside assistance, as needed.

There are a wide variety of methods for personnel certification being used today, with varying degrees of credibility in the marketplace. These programs can be broken into three main categories:

- $\quad$ Registry - A list of people who have met pre-defined qualifications

$\circ$ Wide variance may exist with individuals on the list as qualifications can be broad

$\circ$ List can become dated if not maintained on an on-going basis

- Certificate - based on a course(s) or training, often with an examination based on the training

- May not have an examination

- Certificate can be for life - no expiration date

- Certification - third party assessment that an individual has obtained the knowledge and skills

○ Based on competencies from a scientific study of the occupation

- Examination is standardized

- Time limited

- Can be revoked through due process

$\circ$ More expensive to develop and maintain; and more expensive to individuals seeking certification

Development of a certification program would include two basic parts: a training program to prepare for the credentialing and the actual professional credentialing under a personnel certification body. 


\section{Preparation/Training for Credentialing}

A training course will be developed for each of the system assessment Standards with each course focusing on how to apply the Standard, and preparation of candidates for the certification exam. The first step in developing the training and the professional exam will be to establish the body of knowledge required to appropriately apply each of the Standards. Once the examination and competency requirements are developed, the course will be finalized. Professional level training will need to be delivered on an on-going basis and updated to keep abreast of changes in technology, measurement techniques, and revisions to the Standards.

\section{Professional Credentialing}

Professional credentialing would include a start-up activity to develop the competency requirements needed for certification. The requirements would likely include the number of years experience in assessing the systems covered by the system assessment Standard and passing an examination.

\section{Additional Assessment Standards}

Additional standards may be developed beyond these four system assessment Standards. Assessment standards on other crosscutting systems, such as fan systems, and process-related standards, such as paint booths, may also be developed.

\section{Value to Industry}

Some industrial firms have already established internal goals to reduce energy consumption and greenhouse gas emissions; participation in SEP offers a practical and transparent approach for achieving and validating these efforts. The value proposition to industrial plants for using the system assessment Standards as part of their overall energy management program includes:

- Provides a structure for identifying system energy efficiency opportunities that address the root causes of system inefficiencies and for making cost-effective recommendations to correct them;

- Provides assurance system assessment recommendations are sound and produce predicted results;

- Increases a company's ability to replicate and document their energy management successes;

- Provides a mechanism for communicating to stakeholders about energy management progress; and

- Provides improved opportunities for utility and state financial incentives for energy efficiency as a result of using recognized assessment practices and $\mathrm{M} \& \mathrm{~V}$ protocols. 


\section{References}

ASME Draft Assessment Standards

ASME EA-1-Draft - Energy Assessment for Process Heating Systems

ASME EA-2-Draft - Energy Assessment for Pumping Systems

ASME EA-3-Draft - Energy Assessment for Steam Systems

ASME EA-4-Draft - Assessment for Compressed Air Systems

McKane, A, and P. Sheaffer. 2008. "System Assessment Standards: Defining the Market for Assessment Services", published in the proceedings of the 30th National Industrial Energy Technology Conference, May 2008, New Orleans, La.

Scheihing, P., Almaguer, J., Schultz, S., McKane, A., Meffert, W. 2009. "Superior Energy Performance: A roadmap for achieving continual energy performance improvement", ACEEE Summer Study on Energy Efficiency in Industry, July 2009, Niagara Falls, New York.

Brown, J., Meffert, W., Ferland, K., Hake, D., Krawczyk, M., Mazza, M., Waz, P. 2009. "Results from the Texas Pilot Project on Manufacturing Plant Energy Efficiency Certification", ACEEE Summer Study on Energy Efficiency in Industry, July 2009, Niagara Falls, New York. 